\title{
ODAMY EXTENDING B2B INTEGRATION TECHNOLOGY ARCHITECTURE
}

\author{
Peter Weiß, Wolffried Stucky \\ Institute for Applied Informatics and Formal Description Methods (AIFB), \\ University of Karlsruhe, 76128 Karlsruhe, GERMANY, \{weiss, stucky\}@aifb,uni-karlsruhe.de
}

\begin{abstract}
In this paper we present an ontology based approach for dynamic integration of business partners on demand. ODAMY, an ontology-based methodology, is proposed allowing flexible partner integration on demand into existing or evolving dynamic business networks. The approach chosen takesfocus on the "softer" but important strategic aspects to measure the strategic fit to discover and select business partners. It provides required mechanisms and methods to support trust building by supporting communication and increases transparency within the network. Network companies are represented by a kind of "fingerprint" describing their co-operation ability and requirements. First, the paper Introduces into the scenario of dynamic business ecosystems. Then developments, trends and requirements of existing b2b integration concepts are discussed. ODAMY is described and the implementation is depicted to demonstrate how the methodology is put into practice.
\end{abstract}

\section{INTRODUCTION}

In the future enterprises will transform themselves into better forms by becoming "more intelligent" [4]. "Intelligent" in this context means ability of a network or company to react and adapt to changing market opportunities and conditions. Thus, a fast and easy docking and quick formation of network entities or new business partners providing needed competencies are vital. [1] discusses the problem of integrating business partners into an existing or with different infrastructures depicting different $b 2 b$ (business-to-business) integration concepts and scenarios. Current b2b integration concepts are mainly based on technical aspects and requirements defining interfaces for information exchange, remote invocation of applications or describing business processes using a shared business vocabulary (recent standards e.g. ebXML, RosettaNet). The strategic aspects are mostly not addressed adequately. This research work aims extending state-of-the-art $b 2 b$ integration technology architectures, dealing with the need to provide criteria to discover, select and integrate business partners on demand into emerging or existing business networks. The chosen approach is to measure the strategic fit of network companies to obtain criteria for the (pre-)selection of business partners. The strategic 
fit is stored as "fingerprint" to supply the underlying infrastructure with strategic selection criteria in machine-processable form ("IT infrastructure layer"). The vision is to support the envisaged self-formation of virtual enterprises within dynamic business ecosystems. The work can be partly related to the discovery and selection of web services from directories.

\section{SCENARIO OF DYNAMIC BUSINESS ECOSYSTEMS}

Before the requirements of existing b2b integration concepts are described, the process of dynamic formation of new business partners is shortly depicted. In figure 1 the typical life-cycle of a virtual organization is shown. ODAMY aims supporting the process of discovery, formation, agreement and operation. The integration process discovers and selects business partners from an open-ended collection of pre-qualified partners is shown. The partners agree to form a pool of potential members of virtual organizations. These potential members are selected from the universe of modules. ${ }^{i}$

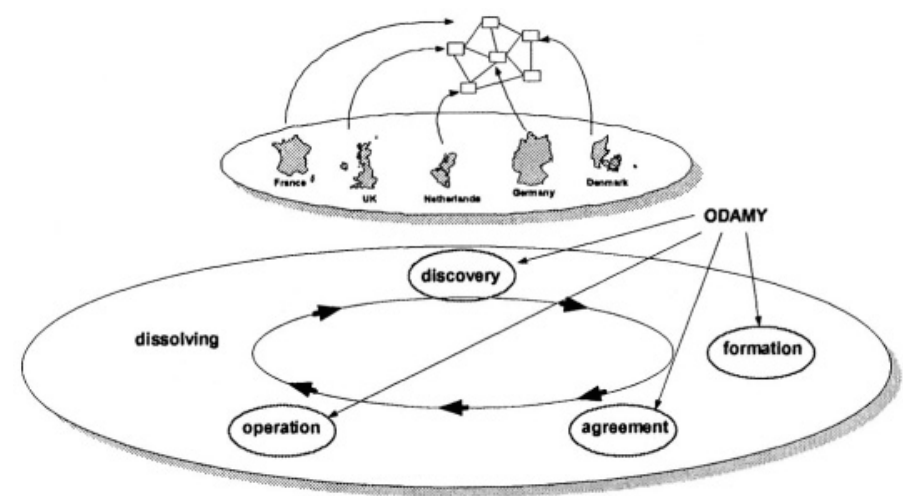

Figure 1 - ODAMY supporting the life-cycle and self-formation of virtual enterprises.

While in the virtual organization, the objective is to exploit a specific market opportunity, the purpose of the "dynamic web" is a disposition to work together in a future market opportunity. This pre-selection of business partners is realized by means of a measurement based on strategic fit criteria. The integration on demand of new partners in such a network is in practice a difficult and time consuming task which runs contrary to the basic idea of flexibility and anticipated fast reactions for virtual organizations. ${ }^{\text {ii }}$ Our experience is that strategic partner fit is a crucial point for success of virtual organizations. Our investigation of virtual organizations determined criteria and dimensions in our opinion essential to measure strategic partner fit and allowing an easy, fast and flexible docking of business partners. We follow a holistic approach which aims providing the underlying IT infrastructure with the strategic selection criteria in machine-processable form. 


\section{B2B INTEGRATION}

Business-to-business (b2b) integration is a buzzword that has been widely used in the past several years and with large variety of meanings. Its meaning ranges from the simple transmission of XML-formatted messages over the Internet to the automated integration of complex dynamic multinational supply chains based on the exchange of electronic business messages, B2b integration is very complex and no common accepted set of integration concepts has been yet developed so far, and no standard architecture is accepted throughout the industrial and research community. In this section we discuss the challenges and needs of a far-out vision of a future business ecosystem based on self-forming virtual enterprises according to [1].

\subsection{Challenges in future business ecosystems}

In the envisaged application scenario of a business ecosystem an enterprise has most of its operations outsourced to service providers or acquires skills needed from business partners. According to [1] a human should only be involved when there is an exceptional case that requires problem-solving skills that cannot be provided in automated form. ${ }^{\text {iii }}$ Therefore, enterprises within a business ecosystem have to announce their capabilities, competencies and skills, indicating to other entities in the network what they could provide. Besides of the important aspects as availability, revenue expectations and cost model, our research takes focus on the need to explicitly represent information regarding goal and risks as well as trust. Trust is the key essence to let business relations run smoothly and automated. Trust is the base for a successful business relationship and therefore vital for the success of business endeavors within self-forming networks. Before engaging, enterprises would like to determine past performance of their potential business partner, including aspects like quality of delivery, flexibility, reliability and dependability. Furthermore, the goals of an enterprise must be clear so that a matchmaking environment can determine if it makes sense to engage. As an enterprise represents a set of individual goals, it is important that there is a fit and to certain degree a good strategic matching with the shared network goals. These goals and the related network culture have to be made explicit, accessible and understandable for human as well as machines. Of course this is still a far-out vision providing a clear direction for research and advanced development. Public registry efforts like UDDI ${ }^{\text {iv }}$ already address and aim solving discovery and selection of services and related service providers [1].

\subsection{Requirements and objectives}

The future exploitation and enormous business potential of new forms of organizations is jeopardized by missing holistic approaches taking into consideration all three layers of organizations (figure 2). The envisaged solution to integrate business partners on demand or initiate and form a collaborative network quickly if the demand occurs has to respond to the requirements of existing b2b integration concepts. Solutions to the scenarios and problems as depicted above can be likely seen as extensions to b2b integration technology architectures with a component that manages the formation of virtual enterprises. That component may use the 
existing b2b integration technology components and add its functionality on top of it [1]. A proper solution has to deliver the information required for the strategic planning, formation and finally operation of business collaborations [1]. A holistic approach has to respond to the needs on the three different layers as depicted in figure 2. The suggested distinction of three layers allows a precise discussion of the goals and focus of $b 2 b$ integration concepts. Today, we observe that most approaches address one or two of the layers but in most cases not a holistic approach. The first layer is the organizational layer. On this level we are dealing with strategic aspects as goals, risks, trust and the network culture. The process layer interacts with the first layer. On this level business processes are described, analyzed and executed. The underlying infrastructure layer is supporting process and organizational layer. Semantics are discussed to overcome the existing barrier between the different levels.

\subsection{Semantic Web}

The currently evolving Semantic Web promises automated information access based on machine-processable semantics of data and heuristics that use these meta data. Within this "new Web", the explicit representation of the semantics of data, accompanied with domain theories (i.e., ontologies), will enable a web that provides a qualitatively new level of service. The Semantic Web heavily relies on formal ontologies that structure underlying data for the purpose of comprehensive and transportable machine understanding. The Semantic Web relies on technologies as $\mathrm{XML}, \mathrm{RDF}, \mathrm{RDF}(\mathrm{S})$ and OWL. ${ }^{\mathrm{V}}$ They properly define the meaning of data and metadata. In general one may consider the Semantic Web more as a vision than a concrete application [7].

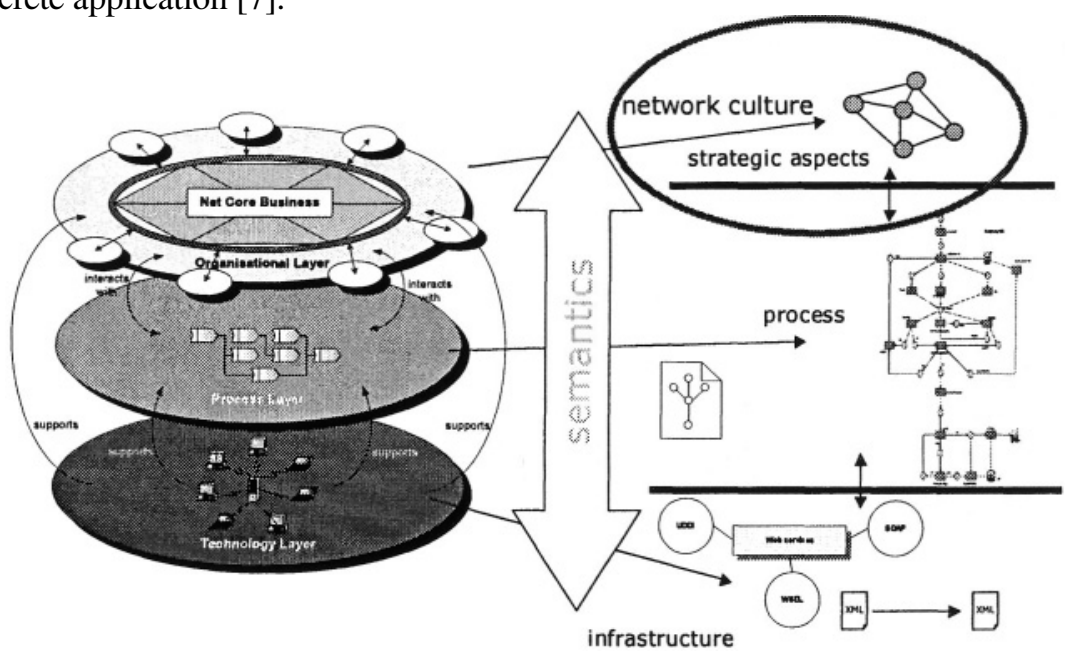

Figure 2-ODAMY extending b2b integration technology architecture.

\subsection{Provision of extended functionality}

Today, most $b 2 b$ integration technology architectures although demanded miss mechanisms and the provision of appropriate functionality to support adequately the 
business relationship management and thus trust building. This constitutes from our point of view a main inhibitor for the further diffusion and application of new forms of organizational and co-operation models. Thus, the core thesis of our research activity is that this inhibitor has to be removed by provision of extended functionality.

\section{ODAMY}

ODAMY aims extending b2b integration technology architecture. The methodology provides criteria to measure the strategic fit of business partners. The information is stored as a fingerprint for each network company in machine-processable form. The semantic web offers required technologies and visions to facilitate the targeted functionality.

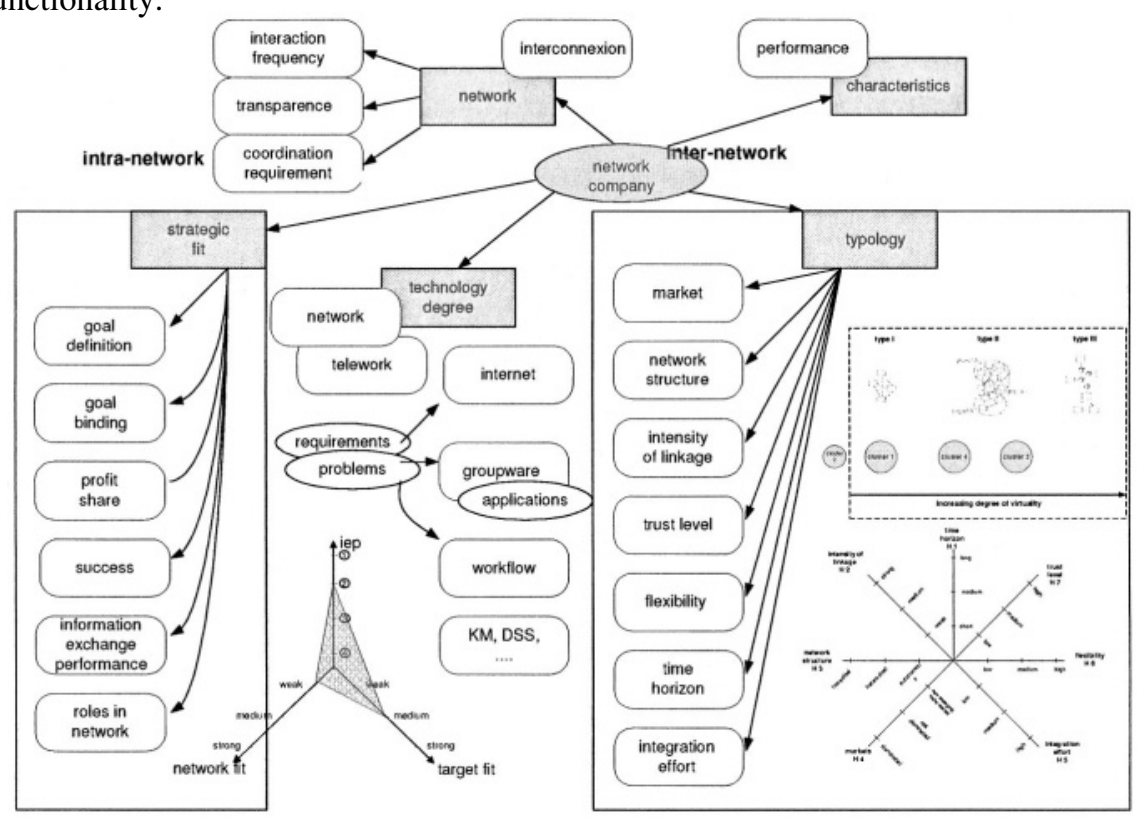

Figure 3: Dimensions to measure network typology and strategic fit.

The goal is to transfer implicit knowledge from the strategic layer (figure 2) into explicit domain knowledge processable by the supporting IT infrastructure layer. The chosen approach is to measure the strategic fit of a network company using an empirical model.

\subsection{Measurement}

An empirical model is applied based on previous research on network typology (inter-net perspective) and strategic partner fit (intra-net perspective) to measure the strategic fit of network companies to create a fingerprint. The criteria for the measurement are the network typology on basis of the assessed virtuality degree 
(seven dimensions) and the strategic fit (three dimensions). Additionally, the created fingerprint of a network company contains information regarding technology degree (usage and experiences with ICT technologies) and related indicators as transparency, interaction frequency and co-operation requirement. Performance indicators may be used to describe past performance. The information is assessed by using an online questionnaire.

\subsection{Fingerprint}

ODAMY methodology [9] combines empirical research and information modeling in form of an ontology-based model. The fingerprint of a network company is produced using a formal explicit ontology-based model structuring criteria for measuring strategic partner fit. In this way, the underlying IT infrastructure is capable for example to infer that a network company "has" a typology based on 'time horizon', 'integration effort', 'intensity of linkage', etc. In figure 4 an excerpt of the fingerprints of two network companies are shown and compared. Company a for example is looking for a co-operation with medium time horizon, whereas the network company $b$ prefers to co-operate in long-term. The companies share the same conceptual model therefore the values are comparable. A matchmaking environment of $\mathrm{a} b 2 \mathrm{~b}$ integration technology architecture can process this information and may have discovered and selected business partners ' $b$ '. The iep of both companies is 'strong', the preferred network structure is 'heterarchical' and the trust-level is 'average', intensity of linkage rated as 'medium' (see figure 3).

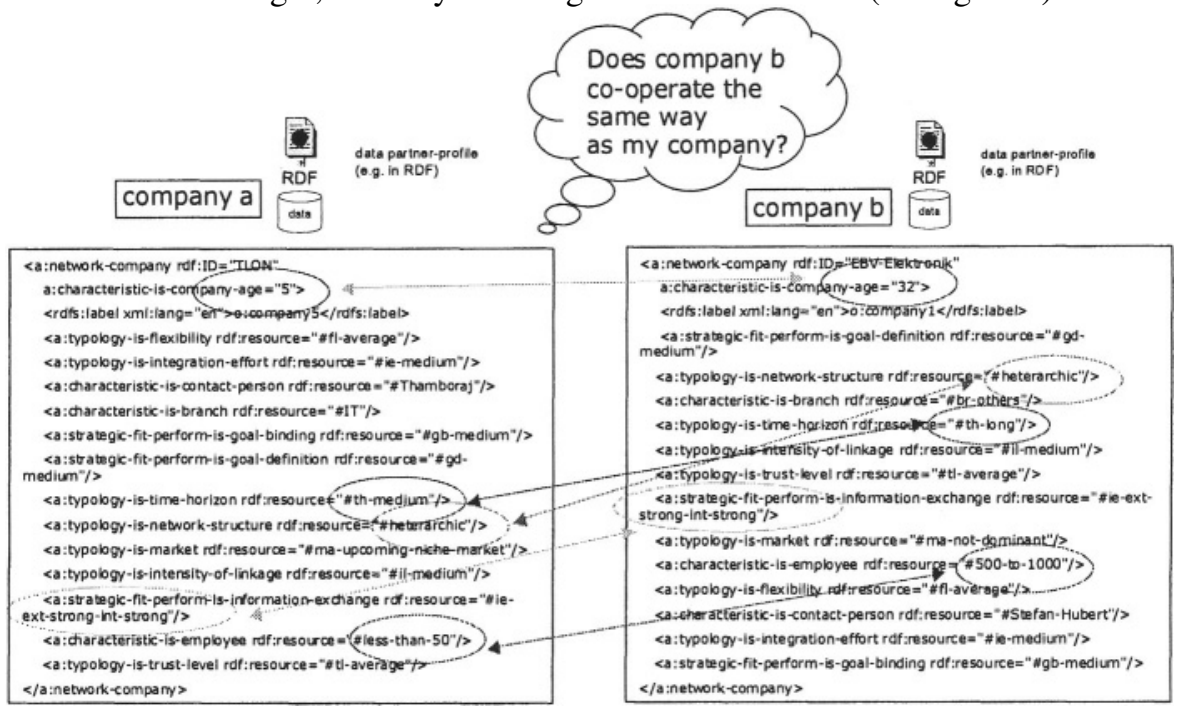

Figure 4 - Excerpt ODAMY fingerprint of network company.

\subsection{Discovery}

Network companies search for potential business partners using the query user interface of the demonstrator (figure 5). As an advantage of using an explicit information model in form of an ontology based model, ODAMY is able to use 
semantic discovery to identify the best fitting partners based on the selected search criteria and applied weightings. The use of ontologies brings service provider and service requestor to a common conceptual space and helps in semantic matching of requirements and specifications. Currently, we are investigating the application of different available semantic discovery mechanisms. The task of matchmaking still requires further investigations and research efforts. However, the Web Ontology Working Group ${ }^{\text {vi }}$ may possibly soon offer an adequate matchmaking environment.

\subsection{Implementation}

ODAMY is implemented using KAON infrastructure [6]. The demonstrator is now available and is currently evaluated using available use cases. A user model was developed defining roles and assigning required tasks to give guidance for the endusers. The user model foresees the role of an information broker who is responsible for the maintenance of the system and the ontology engineering. The end-user is a network company who has to register to create a fingerprint to be stored in the directory. The information broker and the network company use the query interface to search for best fitting partners based on the chosen criteria and possible weightings to express the importance for each criterion. By this means, the network company with the highest strategic partner fit can be discovered and selected.

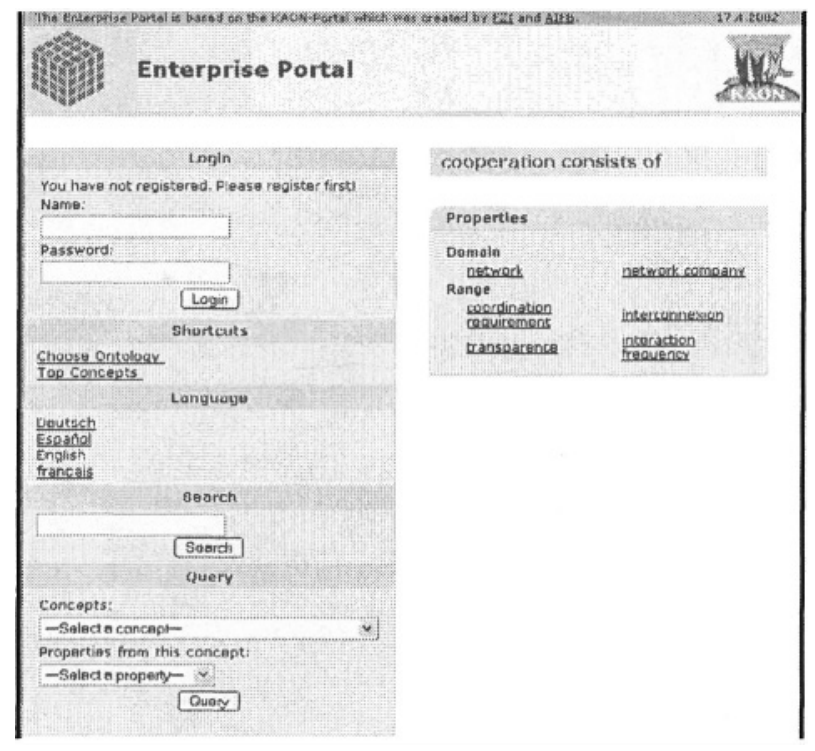

Figure 5 - ODAMY demonstrator.

\section{CONCLUSIONS}

In this paper we presented ODAMY to implement an efficient partner relationship management in dynamic business ecosystems. The methodology intends to extent existing $b 2 b$ integration technology architectures. ODAMY transforms the metrics 
of an empirical model into an ontology based model. KAON tool set and infrastructure is used to implement and demonstrate its functionality. In the future we will have to pay further attention on how to integrate ODAMY in the concrete working process. Our future research activities will concentrate on its extension by using a decentralized model for the fingerprints.

\section{REFERENCES}

1. Bussler, C., B2B Integration, Springer, 2003

2. Camarinha-Matos, L.M., IFIP TC5/WG5.5, Proceedings PRO-VE'03 Proceedings, 11. Processes and foundations for virtual organizations, Kluwer Academic Publishers.

3. Camarinha-Matos, L.M., IFIP TC5/WG5.5, Proceedings $3^{\text {rd }}$ Working Conference on Infrastructures for Virtual Enterprises (PRO-VE'02, Kluwer Academic Publishers, 2002.

4. Delic, K.A.; Dayal U.; The Rise of the Intelligent Enterprise. In: Virtual strategist (2003), Spring, Nr. 5 - A journal of strategy \& business transformation. This article originally appeared in Ubiquity, published online by the Association of Computing Machinery at http://www.acm.org/ubiquity.

5. Franke U., Hickmann, B. Is the Net-Broker an Entrepreneur? What Role does the Net-Broker play in Virtual Webs and Virtual Corporations? Proceedings of the 2nd International VoNet Workshop, Zurich, September 23-24, 1999.

6. KAON - The Karlsruhe Ontology and Semantic Web Framework. Developer's Guide for KAON 1.2.5, February 2003, http://wim.fzi.de.

7. Maedche, A., Weiß, P., Towards Ontology-based Smart Organizations, PRO-VE' 02, Conference Proceedings, 3rd IFIP Working Conference on Infrastructures for Virtual Enterprises, 2. May 2002, Sesimbra, Portugal;

8. Maedche, A.; Ontology learning for the semantic web, Fakultät für Wirtschaftswissenschaften der Universität Karlsruhe (TH), Dissertation 2001, Kluwer.

9. Weiß P., Maedche, A., Towards adaptive ontology-based business networks, , PRO-VE'03 Proceedings, 4th IFIP Working Conference on Virtual Enterprises, 30 October 2003, Lugano, Switzerland

10. Weiß, P., Set up and management of SME business networks, eBusiness and eWork 2002, e2002 Proceedings, 16. October 2002, Prague, Czech Republic;

11. Weiß P., Trunko, R., Smart organization metrics - partner fit, PRO-VE' 02, Conference Proceedings, 3rd IFIP Working Conference on Infrastructures for Virtual Enterprises, 2. May 2002, Sesimbra, Portugal;

\footnotetext{
${ }^{i}$ The universe of modules is the set of all organizations and is composed of the individual organizations of, for example, a specific industry or economy.

ii The transaction cost theory is manifesting this statement arguing the need to reduce efforts to find, select, negotiate and integrate the right business partners on demand.

iii The automated detection of business partners with needed skills, competencies or required technologies as well as their contracting is called self-forming.

${ }^{\text {iv }}$ A public registry is a publicly accessible repository or database where advertising enterprises can store their interface processes. Since these registries are public, any discovering enterprise can access and search them. An example is UDDI, please visit http://www.uddi.org.

${ }^{v}$ For further information please visit http://www.w3.org/2001/sw/.

${ }^{\text {vi }}$ Further information can be obtained from the "Web Ontology Working Group" visiting http://www.w3.org/2001/sw/WebOnt/.
} 